\title{
Chapter 76 \\ Report on the Global Environment \\ Competitiveness of Kazakhstan
}

Kazakhstan across Asia and Europe, including the north of central Asia and the southwest of Ural which is in the Eastern Europe. It faces the Caspian sea, borders on Russia, China, Uzbekistan, Kyrgyzstan and other countries. Its continental climate. The uranium, copper, lead, zinc and chromium are rich. It covers 2,700 thousand square kilometres. It had a population of 16.5 million and domestic production the gross (GDP) of USD 188 billion in 2011. Through the evaluation of global environment competitiveness, we can know that the environment competitiveness index of Kazakhstan ranks at 128 among 133 countries.

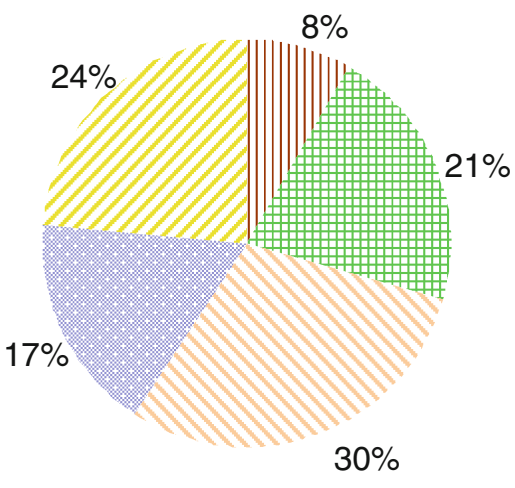

III Resource Environment Competitiveness

\#\# Ecological Environment Competitiveness

Environment Carrying Competitiveness

(l. Environment Management Competitiveness Environment

Fig. 76.1 Contribution of $30 \%$ Harmony Competitiveness 


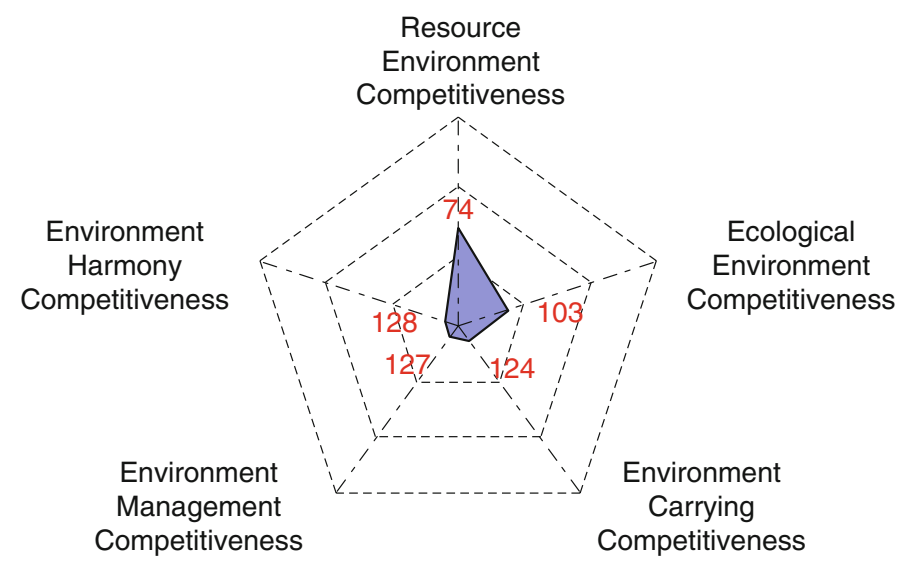

Fig. 76.2 Rank of sub-index of GEC

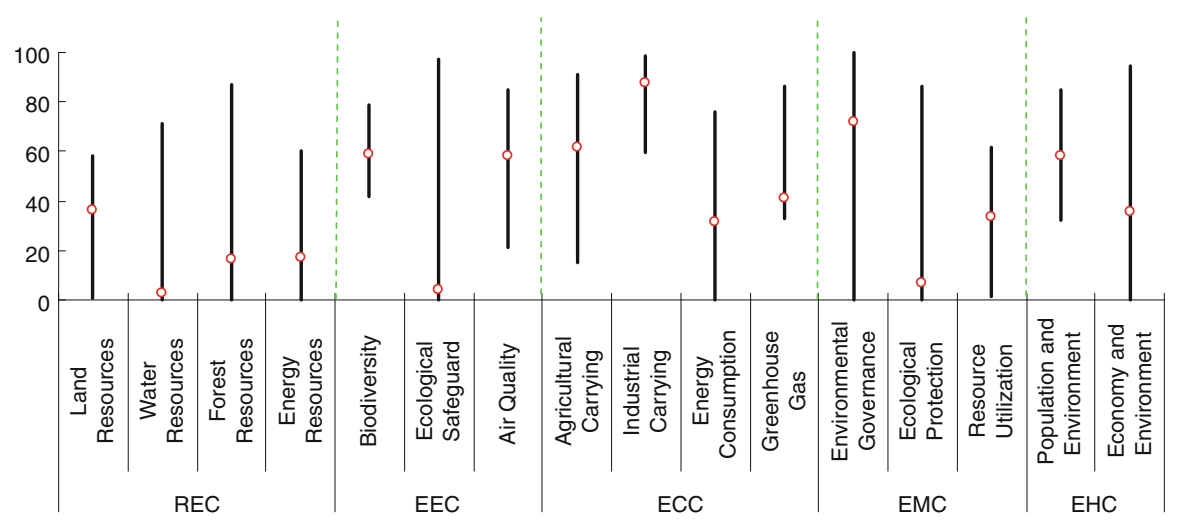

Fig. 76.3 Score and rank of the pillars of GEC

Table 76.1 Score and rank of all indicators of GEC

\begin{tabular}{|c|c|c|c|c|c|}
\hline Indicators & Score & Rank & Indicators & Score & Rank \\
\hline 1 Resource Environment & 16.67 & 74 & Groundwater & 1.76 & 104 \\
\hline Competitiveness & & & Total internal renewable water & 1.20 & 116 \\
\hline 1.1 Land Resources & 36.63 & 6 & resources & & \\
\hline Land area per capita & 29.37 & 9 & 1.3 Forest Resources & 16.32 & 114 \\
\hline $\begin{array}{l}\text { Percentage of arable land to } \\
\text { total land area }\end{array}$ & 14.97 & 87 & $\begin{array}{l}\text { Growing stock in forest and } \\
\text { other wooded land }\end{array}$ & 51.10 & 71 \\
\hline Arable land per capita & 67.96 & 2 & Proportion of land area & 1.43 & 120 \\
\hline 1.2 Water Resources & 3.01 & 116 & covered by forest & & \\
\hline Surface water & 0.51 & 112 & Forest area per capita & 1.39 & 73 \\
\hline Annual precipitation & 8.56 & 113 & 1.4 Energy Resources & 17.26 & 23 \\
\hline
\end{tabular}


Table 76.1 (continued)

\begin{tabular}{|c|c|c|c|c|c|}
\hline Indicators & Score & Rank & Indicators & Score & Rank \\
\hline Fossil energy & 32.84 & 5 & 3.3 Energy Consumption & 31.37 & 99 \\
\hline Energy production & 9.70 & 11 & Energy consumption per unit & 99.94 & 29 \\
\hline Proportion of combustible & 0.07 & 114 & of land area & & \\
\hline $\begin{array}{l}\text { renewables and waste to } \\
\text { total energy consumption }\end{array}$ & & & $\begin{array}{l}\text { Ratio of clean energy } \\
\text { consumption }\end{array}$ & 1.53 & 91 \\
\hline $\begin{array}{l}\text { Net energy imports of the } \\
\text { energy consumption }\end{array}$ & 26.68 & 23 & $\begin{array}{l}\text { Elasticity of energy } \\
\text { consumption }\end{array}$ & 13.29 & 101 \\
\hline $\begin{array}{l}2 \text { Ecological Environment } \\
\text { Competitiveness }\end{array}$ & 41.98 & 103 & $\begin{array}{l}\text { Elasticity of electric power } \\
\text { consumption }\end{array}$ & 10.70 & 63 \\
\hline 2.1 Biodiversity & 58.79 & 39 & 3.4 Greenhouse Gas & 41.21 & 129 \\
\hline Threatened fish species & 93.40 & 41 & Growth rate of $\mathrm{CO}_{2}$ emissions & 20.23 & 129 \\
\hline Threatened mammal species & 91.30 & 90 & Growth rate of Methane & 50.40 & 111 \\
\hline Threatened plant species & 99.07 & 74 & emissions & & \\
\hline $\begin{array}{l}\text { GEF benefits index for } \\
\text { biodiversity }\end{array}$ & 5.10 & 47 & $\begin{array}{l}\mathrm{CO}_{2} \text { emissions per unit of } \\
\text { land area }\end{array}$ & 99.90 & 48 \\
\hline 2.2 Ecological Safeguard & 3.91 & 115 & $\mathrm{CO}_{2}$ emissions per unit of & 15.29 & 123 \\
\hline Terrestrial protected areas & 6.52 & 109 & energy consumption & & \\
\hline Marine protected areas & N/A & N/A & 4 Environment Management & 34.38 & 127 \\
\hline 2.3 Air Quality & 57.92 & 68 & Competitiveness & & \\
\hline Inhalable particles (PM10) & 86.86 & 30 & 4.1 Environmental & 71.94 & 104 \\
\hline Particulate matter (PM2.5) & 83.38 & 75 & Governance & & \\
\hline Index of indoor air pollution & 30.60 & 64 & Agricultural chemicals & 38.10 & 82 \\
\hline Nitrogen oxides emission & 65.25 & 98 & & & 60 \\
\hline Sulfur dioxide emission & 32.71 & 125 & $\begin{array}{l}\text { ercentage of the rural } \\
\text { population with access to }\end{array}$ & 90.00 & 00 \\
\hline $\begin{array}{l}3 \text { Environment Carrying } \\
\text { Competitiveness }\end{array}$ & 59.21 & 124 & an improved water source & & \\
\hline 3.1 Agricultural Carrying & 61.63 & 107 & Percentage of the urban & 99.00 & 51 \\
\hline $\begin{array}{l}\text { Cereal yield per unit of arable } \\
\text { land }\end{array}$ & 4.87 & 123 & an improved water source & & \\
\hline $\begin{array}{l}\text { Fertilizer consumption per } \\
\quad \text { unit of arable land }\end{array}$ & 99.81 & 9 & $\begin{array}{l}\text { 4.2 Ecological Protection } \\
\text { Area of plantation and } \\
\text { afforestation }\end{array}$ & $\begin{array}{l}6.75 \\
1.17\end{array}$ & $\begin{array}{r}125 \\
37\end{array}$ \\
\hline Annual freshwater & 99.11 & 60 & Biome protect & 14.20 & 111 \\
\hline $\begin{array}{l}\text { withdrawals for } \\
\text { agriculture per unit of } \\
\text { arable land }\end{array}$ & & & $\begin{array}{l}\text { Overfishing of fishing } \\
\text { resources }\end{array}$ & N/A & N/A \\
\hline 3.2 Industrial Carrying & 87.35 & 99 & 4.3 Resource Utilization & 33.65 & 78 \\
\hline $\begin{array}{l}\text { Net exports as a percentage of } \\
\text { GDP }\end{array}$ & 70.06 & 101 & $\begin{array}{l}\text { Utilization rate of water } \\
\text { resources }\end{array}$ & 1.17 & 30 \\
\hline $\begin{array}{l}\text { Electric power consumption } \\
\text { per unit of value added of } \\
\text { industry }\end{array}$ & 90.87 & 71 & $\begin{array}{l}\text { Percentage of total internal } \\
\text { renewable water resources } \\
\text { to total water resources }\end{array}$ & 39.86 & 98 \\
\hline $\begin{array}{l}\mathrm{SO}_{2} \text { emissions per unit of } \\
\text { value added of industry }\end{array}$ & 98.72 & 122 & $\begin{array}{l}\text { Percentage of agricultural } \\
\text { land to total land area }\end{array}$ & 91.28 & 5 \\
\hline $\begin{array}{l}\text { Annual freshwater } \\
\text { withdrawals for industry } \\
\text { per value added of } \\
\text { industry }\end{array}$ & 89.75 & 115 & $\begin{array}{l}\text { Percentage of fossil fuel } \\
\text { energy consumption } \\
\text { to total energy } \\
\text { consumption }\end{array}$ & 2.29 & 116 \\
\hline
\end{tabular}


Table 76.1 (continued)

\begin{tabular}{|c|c|c|c|c|c|}
\hline Indicators & Score & Rank & Indicators & Score & Rank \\
\hline $\begin{array}{l}5 \text { Environment Harmony } \\
\text { Competitiveness }\end{array}$ & 46.94 & 128 & $\begin{array}{l}\mathrm{CO}_{2} \text { emissions (metric tons } \\
\text { per capita) }\end{array}$ & 61.51 & 123 \\
\hline $\begin{array}{l}\text { 5.1 Population and } \\
\text { Environment }\end{array}$ & 58.36 & 121 & $\begin{array}{l}\text { Energy consumption per } \\
\text { capita }\end{array}$ & 64.82 & 109 \\
\hline \multirow{2}{*}{$\begin{array}{l}\text { Improved sanitation facilities } \\
\text { (\% of population with } \\
\text { access) }\end{array}$} & 97.00 & 39 & $\begin{array}{l}5.2 \text { Economy and } \\
\text { Environment }\end{array}$ & 35.52 & 124 \\
\hline & 75.68 & 82 & $\begin{array}{l}\text { Land resource utilization } \\
\text { efficiency }\end{array}$ & 0.02 & 105 \\
\hline $\begin{array}{l}\text { Motor vehicles (per 1,000 } \\
\text { people) }\end{array}$ & 4.70 & 55 & $\begin{array}{l}\text { Sulfur dioxide emissions per } \\
\text { unit of GDP }\end{array}$ & 39.44 & 126 \\
\hline \multirow{2}{*}{$\begin{array}{l}\text { Renewable internal freshwater } \\
\text { resources per capita } \\
\mathrm{SO}_{2} \text { emissions (metric tons } \\
\text { per capita) }\end{array}$} & 27.82 & 128 & $\begin{array}{l}\text { Carbon dioxide emissions per } \\
\text { unit of GDP }\end{array}$ & 39.51 & 128 \\
\hline & & & $\begin{array}{l}\text { Energy consumption per unit } \\
\text { of GDP }\end{array}$ & 63.11 & 112 \\
\hline
\end{tabular}

Table 76.2 Rank distribution of the individual indicators of GEC

\begin{tabular}{lllllll}
\hline & $\begin{array}{l}\text { Number } \\
\text { of the } \\
\text { individual }\end{array}$ & Rank & Rank & Rank & Rank & Rank \\
indicators & $1-10$ & $11-30$ & $31-60$ & $61-100$ & $101-133$ \\
\hline Sub-index & 14 & 3 & 2 & 0 & 2 & 7 \\
\hline $\begin{array}{l}\text { Resource Environment } \\
\quad \text { Competitiveness }\end{array}$ & 11 & 0 & 1 & 2 & 6 & 2 \\
$\begin{array}{c}\text { Ecological Environment } \\
\quad \text { Competitiveness } \\
\begin{array}{c}\text { Environment Carrying } \\
\quad \text { Competitiveness }\end{array}\end{array}$ & 15 & 1 & 1 & 1 & 4 & 8 \\
$\begin{array}{c}\text { Environment Management } \\
\quad \text { Competitiveness }\end{array}$ & 10 & 1 & 1 & 2 & 3 & 3 \\
$\begin{array}{c}\text { Environment Harmony } \\
\text { Competitiveness }\end{array}$ & 10 & 0 & 0 & 2 & 1 & 7 \\
Total & 60 & 5 & 5 & 7 & 16 & 27 \\
\hline
\end{tabular}

Open Access This chapter is distributed under the terms of the Creative Commons Attribution Noncommercial License, which permits any noncommercial use, distribution, and reproduction in any medium, provided the original author(s) and source are credited. 